\title{
ANALYSIS OF A 4-SPAN CONTINUOUS PLATE IN ONE DIRECTION USING MATLAB PROGRAMMING
}

\author{
ADAH E. I., UBI S. E., IDAGU F. O. AND UBI P. A.
}

(Received 18 February 2021; Revision Accepted 10 March 2021)

\begin{abstract}
The present study presents a computer approach based on polynomial shape functions application for analysis of continuous plate in one direction using Matlab. A 4-span continuous plate in the x-direction comprises of boundary conditions SSSC, SCSC, SCSC and SCSS single panels' plate was analyzed. It was assumed that, the external edges were simply supported while the internal edges of each panel were clamped. The bending moments of the clamped edges were calculated for each panel using appropriate boundary conditions which formed the fixed end moments (FEMs). Stiffness method was used based on beam analogy to analyze the continuous plate. Matlab codes were applied to develop a computer program for this analysis. To validate the results of this present study, the values of the moments obtained were compared with those of earlier studies using manual method. The percentage difference for fixed end moments were all $0.00 \%$ and that of support moments had the maximum value of $0.016 \%$. Thus, it was concluded that the present study program based on Matlab is adequate and a faster approach for a 4span continuous plate in one direction analysis.
\end{abstract}

KEYWORDS: Matlab Programming, Continuous Plate, Polynomial Shape Functions, Beam Analogy, Fixed Edge Moment.

\section{SYMBOLS}

$\mathrm{w}=$ Deflection; $\mathrm{v}=$ Poisson Ratio

$\mathrm{r}=$ Non dimensional Parameter in $\mathrm{X}$ direction $(=\mathrm{X} / \mathrm{a})$; $\mathrm{q}=$ Non dimensional Parameter in $\mathrm{Y}$ direction $(=\mathrm{Y} / \mathrm{b})$

$\mathrm{U}, \mathrm{V}=$ Deflection parts in $\mathrm{X} \& \mathrm{Y}$ Directions for Non-dimensional Parameters

$\mathrm{Z}=$ Aspect Ratio $(=\mathrm{b} / \mathrm{a}) ; \mathrm{a}=$ dimension along $\mathrm{x}$ direction; $\mathrm{b}=$ dimension along y direction

$\mathrm{u}_{\mathrm{s}}=$ Coefficient of amplitude in terms of $\mathrm{S}$;

$\beta$ or beta $=$ Moment Coefficient considered at the edge along $r$ - direction

$\mathrm{w}^{\prime \prime} \mathrm{R}=\frac{\partial^{2} \mathrm{w}}{\partial R^{2}} ; \quad \mathrm{w}^{\prime \prime \mathrm{Q}}=\frac{\partial^{2} \mathrm{w}}{\partial Q^{2}} ; \mathrm{h}^{\prime \prime} \mathrm{R}=\quad \frac{\partial^{2} \mathrm{~h}}{\partial R^{2}} ; \mathrm{h}^{\prime \prime} \mathrm{Q}=\frac{\partial^{2} \mathrm{~h}}{\partial Q^{2}}$

$\mathrm{Q}=\mathrm{Udl}, \mathrm{M}_{\mathrm{xe}}$, = Edge Moment in $\mathrm{X}$, fem = fixed end moment

mavs $=$ final support moment, mspan $=$ span moment

\section{INTRODUCTION}

Continuous plates are externally statically indeterminate. Force and deformation methods are the usual classical methods used in the analysis of continuous plates. Example of these plates are floor slabs used in buildings and bridges, sheet walls, etc. These are usually supported by exterior walls and often have intermediate supports in the form of beams and partitions or columns (Timoshenko and Woinowsky-Krieger, 1959; Ventsel and Krauthamer, 2001; Szilard, 2004). Classical analysis of continuous plate with exception of the simplest cases is quite cumbersome (Szilard, 2004).
Again, the application of the rigorous classic methods to the design of continuous plate floor slabs often leads to cumbersome calculations and the accuracy this obtained is illusory on account of many more or less indeterminable factors affecting the magnitude of the moments of the plate (Timoshenko and WoinowskyKrieger, 1959). Numerical approaches are used almost exclusively to obtain usable approximate solutions of these important plate problems in an economical way (Szilard, 2004, Bakhaty et al. 2016, Chalal, 2017, Yang et al. 2018). Due to the complexity of using trigonometric shape function. Ibearugbulem et al. (2014), Onwuka et al. (2016), and Adah (2016), had earlier applied

Adah, E. I., Department of Civil and Environmental Engineering, University of Calabar, Nigeria

Ubi, S. E., Department of Civil Engineering, Cross River University of Technology, Calabar, Nigeria

Idagu, F. O., Department of Civil Engineering, Federal University of Technology, Owerri, Nigeria

Ubi, P. A., Department of Mechanical Engineering, University of Calabar, Nigeria 
polynomial shape functions to single panel plate analysis. Furthermore, Adah et al. (2020), Adah et al. (2021), used polynomial shape functions as a new approach in analysis of continuous plate in both single direction and two directions and the results so obtained were adequate. Even though much relief from cumbersome calculations was achieved, the approach is still a little bit time consuming.

Matlab is a powerful mathematical-based software which is used widely in programming engineering problems
(Wilson, et al. 2003; Yang, et al. 2005). It is worth mentioning that, none of the researchers have developed Matlab programs using polynomial shape functions in continuous plate analysis. Thus, the aim of this work was to simplify this problem by the development of Matlab program using polynomial shape functions to obtain easily the fixed end moment and support moments of the plates.

\section{FUNDAMENTAL EQUATIONS}

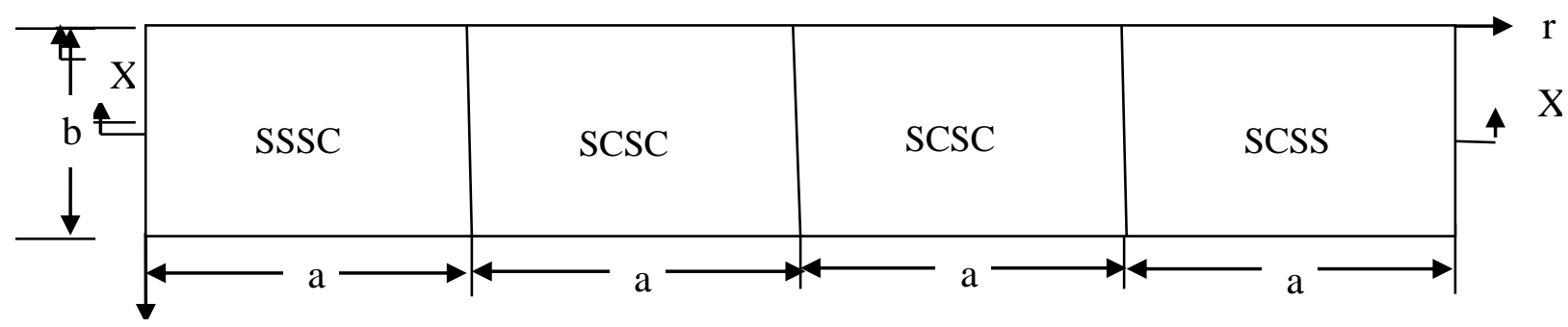

q

Figure 1: Schematic Representation of a Continuous Plate

Adah (2016) gave the general deflected shape function for rectangular isotropic plates as

$w=w^{R *} w^{Q}=A\left(c_{1} R^{f 1}-d_{1} R^{m 1}+e_{1} R^{n 1}\right)\left(c_{2} Q_{1}^{f 2}-d_{2} Q^{m 2}+e_{2} Q^{n 2}\right)$

where $c_{1}, c_{2}, f^{1}, f^{2}, d_{1}, d_{2}, m^{1}, m^{2}, e_{1}, e_{2}$ and $n^{1}, n^{2}$ are coefficients.

and $h=\left(c_{1} R^{f 1}-d_{1} R^{m 1}+e_{1} R^{n 1}\right)\left(c_{2} Q^{f 2}-d_{2} Q^{m 2}+e_{2} Q^{n 2}\right)=U V$

If $R=r$ and $Q=q$; then, the shape profile of the three boundary conditions in Figure 1 are given by Adah (2016) as equations 3-5.

For SSSC; $h=\left(0.5 r-1.5 r^{3}+r^{4}\right)\left(q-2 q^{3}+q^{4}\right)(3)$

For SCSC; $h=\left(r^{2}-2 r^{3}+r^{4}\right)\left(q-2 q^{3}+q^{4}\right)$

For SCSS; $h=\left(1.5 r-2.5 r^{3}+r^{4}\right)\left(q-2 q^{3}+q^{4}\right)(5)$

Where $S$ is simply supported edge and $C$ is clamped edge of the plate.

Adah (2016) gave the bending moment of a rectangular isotropic plate in both $\mathrm{x}$ - direction as

$\mathrm{M}_{\mathrm{x}}=-\mathrm{u}_{\mathrm{s}} \mathrm{qa} \mathrm{a}^{2}\left(\frac{\partial^{2} \mathrm{~h}}{\partial R^{2}}+\mathrm{v} \frac{\partial^{2} \mathrm{~h}}{2^{2} \partial Q^{2}}\right)$

let $\beta=-u_{s}\left(\frac{\partial^{2} h}{\partial R^{2}}+v \frac{\partial^{2} h}{2^{2} \partial Q^{2}}\right)$

Then,

$\mathrm{M}_{\mathrm{x}}=\beta q a^{2}$

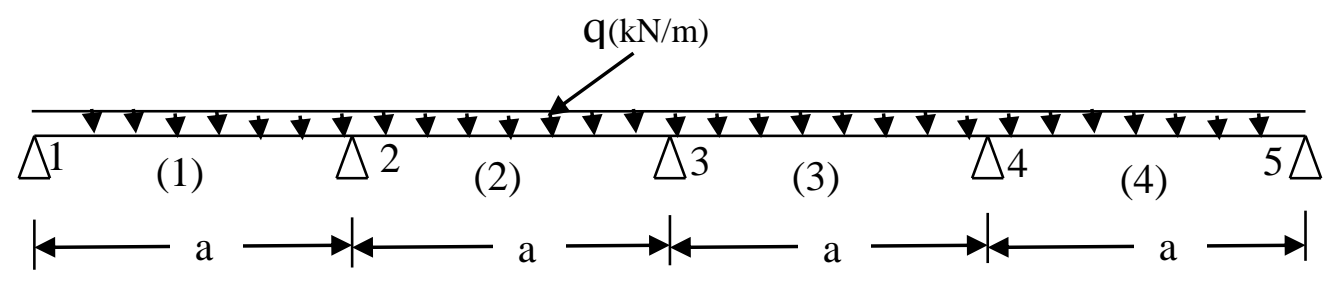

Fig 2a: Section X-X, showing a plate strip loaded uniformly

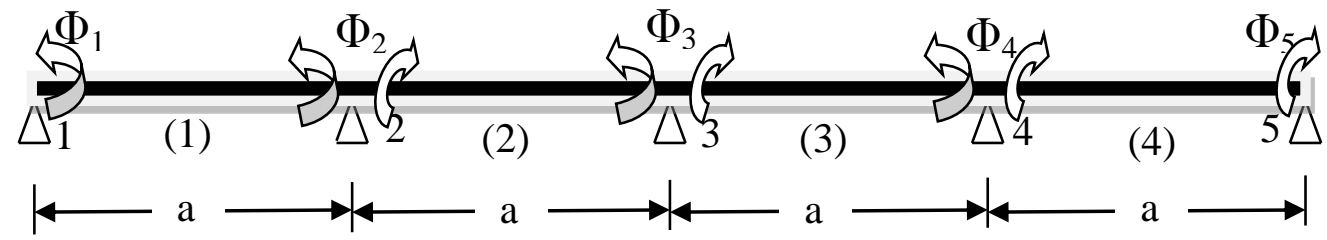

Fig 2b: Section X-X, showing the supports rotations. Take EI as constant 
From Figure 1, a section $\mathrm{X}-\mathrm{X}$ along the longitudinal axis was taken and analyzed (Figure 2.0). It was assumed that the external edges were simply supported while the internal edges were clamped. By using beam analogy, a section $X-X$ through the center of each panel was then analyzed using stiffness method for support and span moments, Adah et al. (2020).

\section{COMPUTER APPLICATION}

Matlab programming codes were applied, the fixed edge moment of each span calculated by substituting the shape profile $\mathrm{h}$, from equations (3) to (5) and the boundary conditions of each single panel into equations (6). The values of the fixed edge moments and support moments of the individual plate obtain are presented in columns 1 and 4 of Table 1.0 respectively. The step by step simplified interactive Matlab program developed is presented in the appendix. The user is expected to know the shape profile of each single panel plate in the continuous plate.

\section{RESULTS AND DISCUSSIONS}

Columns 1 and 4 of Table one shows the coefficients of FEMs and SM obtained from this work. In order to validate the results obtained from this present program, the values of the fixed end moments (FEMs) and support moments (SM) were compared with those of earlier studies as presented in Table 1.0. The percentage difference for FEMs were $0.00 \%$. This indicated that there was no difference in the results. Also, the percentage difference for the SM had a maximum of $0.016 \%$. This is far less than $5 \%$ and was deemed satisfactory. Therefore, the conclusion that this present study program is satisfactory and provides a faster approach for a 4-span continuous plate analysis in one direction

Table 1.0: Values of $\beta_{1} \& \beta_{3}$ from manual Method and $\beta_{2}$ and $\beta 4$ from PROGRAM for Continuous plate in One direction. $2=1,4$ spans

\begin{tabular}{|c|c|c|c|c|c|}
\hline $\begin{array}{l}\text { Present study } \\
\text { FEM }=\beta q a^{2} \beta_{1}\end{array}$ & $\begin{array}{c}\text { Adah et al. } 2020 \\
\qquad \begin{array}{c}\text { FEM }=\beta q^{2} \\
\beta_{2}\end{array}\end{array}$ & $\begin{array}{c}\text { Percentage } \\
\text { Difference } \\
100\left(\beta_{2}-\beta_{1}\right) / \beta_{1}\end{array}$ & $\begin{array}{c}\text { Present study } \\
\qquad \begin{array}{c}S M=\beta q a^{2} \\
\beta_{3}\end{array}\end{array}$ & $\begin{array}{c}\text { Adah et al. } 2020 \\
S M=\beta q a^{2} \\
\beta_{4}\end{array}$ & $\begin{array}{c}\text { Percentage } \\
\text { Difference } \\
100\left(\beta_{4}-\beta_{3}\right) / \beta_{3}\end{array}$ \\
\hline 0.0000 & 0.0000 & 0.00 & 0.00000 & 0.00000 & 0.00 \\
\hline-0.06760 & -0.06760 & 0.00 & -0.06590 & -0.06590 & 0.00 \\
\hline-0.06363 & -0.06363 & 0.00 & -0.06136 & -0.06135 & 0.016 \\
\hline-0.06363 & -0.06363 & 0.00 & -0.06250 & -0.06249 & 0.016 \\
\hline-0.06363 & -0.06363 & 0.00 & -0.06476 & -0.06476 & 0.00 \\
\hline-0.06363 & -0.06363 & 0.00 & -0.06590 & -0.06590 & 0.00 \\
\hline-0.06760 & -0.06760 & 0.00 & -0.06930 & -0.06930 & 0.00 \\
\hline 0.0000 & -0.00000 & 0.00 & 0.00000 & 0.00000 & 0.00 \\
\hline
\end{tabular}

\section{CONCLUSION}

The results of this present study program for a 4-span continuous plates analysis in one direction using polynomial shape functions was proven to be adequate. Thus, it could be concluded that the present

\section{REFERENCE}

Adah, E. I., 2016. Development of Computer Programs for the Analysis of Single Panel and Continuous Rectangular Plate, M. Eng. Thesis submitted to postgraduate school, Federal University of Technology, Owerri.

Adah, E. I., Onwuka, D. O., and Ibearugbulem, O. M., 2016. Development of polynomial-based program for pure bending analysis of SSSS rectangular thin isotropic plate, International Journal of science, engineering and technological research, 5(7): 2290-2295. study program using polynomial shape functions was adequate and a more faster approach for a 4-span continuous plate in one direction analysis.

Adah, E. I., Ibearugbulem, O. M., and Ubi, P. A. 2020. Analysis of continuous plate using polynomial displacement functions based on beam analogy, Umudike Journal of Engineering and Technology, 6 (1): 111-119.

Adah, E. I., Ibearugbulem, O. M., Ubi, S. E., and Idagu, F. O., 2021. Analysis of a two-way continuous plate based on beam analogy. ISOR Journal of Mechanical and Civil Engineering, 18(1), 59-67.

Bakhaty, A. A, Govindjee, S., and Mosalam, K, M. 2016. Theoretical evaluation of hybrid simulation applied to continuous plate structures, ASCE Journal of Eng. Mech., 142(12). 
Chalal, F. Z. Optimum design of continuous plate girder with variable depth, 2017, 8(3); 494-509.

Ibearugbulem, O. M., Ezeh J. C., and Ettu, L. O., 2014. Energy methods in theory of rectangular plates: Use of polynomial Shape Functions, Owerri, Liu House of excellence ventures,.

Onwuka, D. O. Ibearugbulem, O. M., and Adah, E. I., 2016. Stability analysis of axially compressed SSSS and CSCS plates using MATLAB programming. International journal of science and technoledge, 4(1); 66-71.

Szilard, R., 2004. Theories and Application of Plate Analysis, New, Jersey, John Wiley and sons.

Timoshenko, S. P., and Woinowsky-Krieger, 1959. Theory of plates and shells, 2nd ed., New York; McGraw Hills.

\section{APPENDIX}

\section{CONTINUOUS PLATE ONE DIRECTION PROGRAM}

clear all

\%CONTINUOUS PLATE IN ONE DIRECTION (XDIRECTION) PROGRAM

$\mathrm{a}=$ input('Enter plate dimension along $\mathrm{x}$-axis -length$\left.\mathrm{a}(\mathrm{m})::^{\prime}\right)$;

$\mathrm{b}=$ input('Enter plate dimension along $\mathrm{y}$-axis -width$\left.\mathrm{b}(\mathrm{m}):^{\prime}\right)$;

$\mathrm{Q}=$ input('Enter the udl $\left.\mathrm{Q}(\mathrm{kN} / \mathrm{m}):^{\prime}\right)$;

$\mathrm{v}=$ input('Enter poision ratio $\mathrm{v}:$ ');

echo on

$\mathrm{s}=\mathrm{b} / \mathrm{a}$

echo off

\%for SSSC span1

syms $r \mathrm{q}$

$U=0.5^{*} r-1.5^{*} r^{\wedge} 3+r^{\wedge} 4$;

$\mathrm{V}=\mathrm{q}-2^{*} \mathrm{q}^{\wedge} 3+\mathrm{q}^{\wedge} 4$

$\operatorname{diff}(\mathrm{U}, 2)$;

$(\operatorname{diff}(U, 2))^{\wedge} 2$;

y1 = int $\left((\operatorname{diff}(U, 2))^{\wedge} 2, r, 0,1\right)$;

$\mathrm{z1}=\operatorname{int}\left(\mathrm{V}^{\wedge} 2, \mathrm{q}, 0,1\right)$;

$\mathrm{Y} 1=\mathrm{y} 1^{\star} \mathrm{z} 1$

$\operatorname{diff}(\mathrm{V}, 2)$;

$(\operatorname{diff}(\mathrm{V}, 2))^{\wedge} 2$

y2 = int $\left(U^{\wedge} 2, r, 0,1\right)$;

$\mathrm{z} 2=\operatorname{int}\left((\operatorname{diff}(\mathrm{V}, 2))^{\wedge} 2, \mathrm{q}, 0,1\right)$;

$\mathrm{Y} 2=\mathrm{y} 2^{*} \mathrm{z} 2$;

$\operatorname{diff}(\mathrm{U}, 1)$;

$\operatorname{diff}(\mathrm{V}, 1)$;

y3 = int $\left((\operatorname{diff}(U, 1))^{\wedge} 2, r, 0,1\right)$;

$\mathrm{z3}=\operatorname{int}\left((\operatorname{diff}(\mathrm{V}, 1))^{\wedge} 2, \mathrm{q}, 0,1\right)$;

$\mathrm{Y} 3=\mathrm{y} 3^{\star} \mathrm{z} 3$

y4 = int $(U, r, 0,1)$;

$\mathrm{z} 4=\operatorname{int}(\mathrm{V}, \mathrm{q}, 0,1)$;

$\mathrm{Y} 4=\mathrm{y} 4^{\star} \mathrm{z} 4$;

$\mathrm{u}=\mathrm{vpa}\left(\mathrm{Y} 4 /\left(\mathrm{Y} 1+\left(2^{*} \mathrm{Y} 3 / \mathrm{s}^{\wedge} 2\right)+\left(\mathrm{Y} 2 / \mathrm{s}^{\wedge} 4\right)\right), 5\right) ;$

$r=$ input('Enter $r$ value at support $2 r: ')$

$\mathrm{q}=$ input('Enter q value at support $2 \mathrm{q}:$ ');

$k=\left(0.5^{\star} r-1.5^{\star} r^{\wedge} 3+r^{\wedge} 4\right)^{*}\left(q-2^{*} q^{\wedge} 3+q^{\wedge} 4\right)$;

echo on

$\%$ Edge moment

echo off

Ventsel, E., and Krauthamer T., 2001. Thin Plates and Shells: Theory, Analysis and Applications, New York, Marcel Dekker.

Wilson, H. B., Turcotte, L. H., and Halpern, D., 2003. Advanced Mathematics and Mechanics Applications using Matlab. 3rd ed, USA, Chapman and Hall/CRC.

Yang, Y.W., Cao, W., Chung, T., and Morries, J., 2005. Applied Numerical Methods using Matlab. New Jersey, John Wiley and Sons, Inc.

Yang, J., Ouyang, H., Stancioiu, D., Cao, S., and He, X. 2018. Dynamic response of a four-span continuous plate structure subjected to moving cars with time-varying speeds, Journal of vibration and acoustic, 140(6).

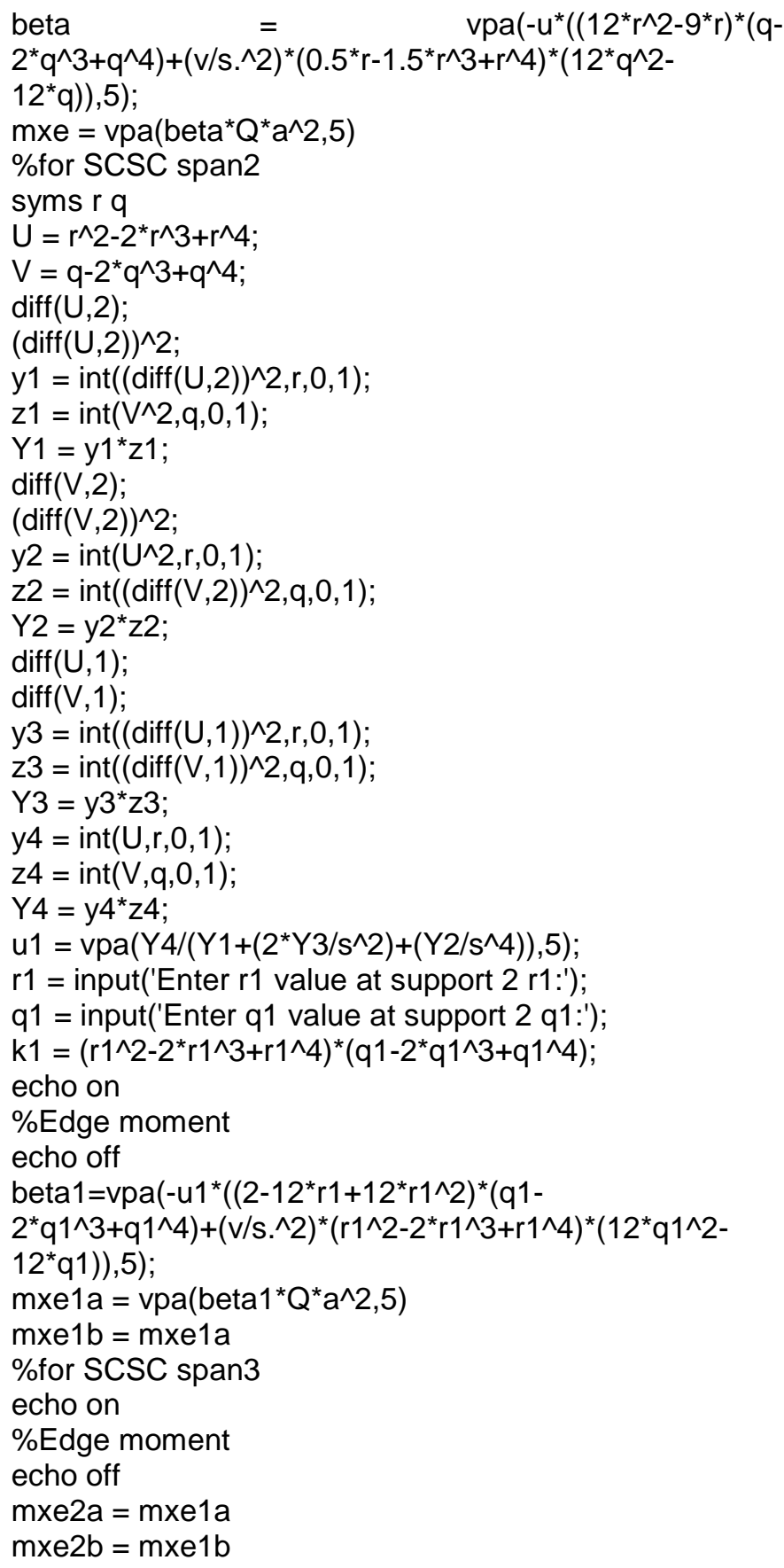


\%for SCSS span4

syms rq

$U=1.5^{\star} r^{\wedge} 2-2.5^{\star} r^{\wedge} 3+r^{\wedge} 4$

$\mathrm{V}=\mathrm{q}-2^{\star} \mathrm{q}^{\wedge} 3+\mathrm{q}^{\wedge} 4$

$\operatorname{diff}(\cup, 2)$;

$(\operatorname{diff}(U, 2))^{\wedge} 2$;

$\mathrm{y} 1=\operatorname{int}\left((\operatorname{diff}(U, 2))^{\wedge} 2, r, 0,1\right)$;

$\mathrm{z} 1=\operatorname{int}\left(\mathrm{V}^{\wedge} 2, \mathrm{q}, 0,1\right)$;

$\mathrm{Y} 1=\mathrm{y} 1^{*} \mathrm{z} 1$;

$\operatorname{diff}(\bigvee, 2)$;

$(\operatorname{diff}(\mathrm{V}, 2))^{\wedge} 2$;

$\mathrm{y} 2=\operatorname{int}\left(\mathrm{U}^{\wedge} 2, \mathrm{r}, 0,1\right)$;

$\mathrm{z} 2=\operatorname{int}\left((\operatorname{diff}(\mathrm{V}, 2))^{\wedge} 2, \mathrm{q}, 0,1\right)$;

$\mathrm{Y} 2=\mathrm{y} 2^{*} \mathrm{z} 2$

$\operatorname{diff}(\bigcup, 1)$;

$\operatorname{diff}(\mathrm{V}, 1)$;

y3 = int $\left((\operatorname{diff}(U, 1))^{\wedge} 2, r, 0,1\right)$;

$\mathrm{z3}=\operatorname{int}\left((\operatorname{diff}(\mathrm{V}, 1))^{\wedge} 2, \mathrm{q}, 0,1\right)$;

$Y 3=y 3^{*} z 3$

y4 = int $(U, r, 0,1)$;

$\mathrm{z} 4=\operatorname{int}(\mathrm{V}, \mathrm{q}, 0,1)$;

$\mathrm{Y} 4=\mathrm{y} 4^{*} \mathrm{z} 4$

$\mathrm{u} 3=\mathrm{vpa}\left(\mathrm{Y} 4 /\left(\mathrm{Y} 1+\left(2^{*} \mathrm{Y} 3 / \mathrm{s}^{\wedge} 2\right)+\left(\mathrm{Y} 2 / \mathrm{s}^{\wedge} 4\right)\right), 5\right)$

r3 = input('Enter r3 value at support 4 r3:');

q3 = input('Enter q3 value at support 4 q3:');

k3 $=\left(1.5^{\star} r 3^{\wedge} 2-2.5^{\star} r 3^{\wedge} 3+r 3^{\wedge} 4\right)^{\star}\left(q 3-2^{*} q 3^{\wedge} 3+q 3^{\wedge} 4\right)$;

echo on

\%Edge moment

echo off

beta3 $=\quad \mathrm{vpa}\left(-\mathrm{u3}{ }^{\star}\left(\left(3-15^{\star} \mathrm{r} 3+12^{*} \mathrm{r} 3^{\wedge} 2\right)^{\star}(\mathrm{q} 3-\right.\right.$

$\left.2^{*} q 3^{\wedge} 3+q 3^{\wedge} 4\right)+\left(v / s .{ }^{\wedge} 2\right)^{*}\left(1.5^{*} r 3^{\wedge} 2-\right.$

$\left.\left.\left.2^{*} r 3^{\wedge} 3+r 3^{\wedge} 4\right)^{*}\left(12^{*} q 3^{\wedge} 2-12^{*} q 3\right)\right), 5\right)$;

$\mathrm{mxe} 3=\operatorname{vpa}\left(\right.$ beta3 $\left.^{\star} \mathrm{Q}^{\star} \mathrm{a}^{\wedge} 2,5\right)$

echo on

\%Fixed end moment at each support(starting from left to right of the plate span)

echo off

$\mathrm{f} 1=0$

$\mathrm{f} 2=\mathrm{mxe}-\mathrm{mxe} 1 \mathrm{a}$

$\mathrm{f} 3=\mathrm{mxe} 1 \mathrm{~b}-\mathrm{mxe} 2 \mathrm{a}$

$\mathrm{f} 4=\mathrm{mxe} 2 \mathrm{~b}-\mathrm{mxe} 3$

$\mathrm{f} 5=0$

\%using element stiffness method of analysis

$\mathrm{E}=$ input('young modulus $\mathrm{E}:$ ');

I = input('second moment of inertia I:')

\%flexural rigidity $(\mathrm{EI})=\mathrm{FR}$

$\mathrm{FR}=\mathrm{E}^{\star} \mathrm{I}$;

$A=[42 ; 24]$;

\%element stiffness

$\mathrm{K} 1=\mathrm{A} .{ }^{*} \mathrm{FR}$;

$\mathrm{K} 2=\mathrm{K} 1$;

$\mathrm{K} 3=\mathrm{K} 1$;

$\mathrm{K} 4=\mathrm{K} 1$;

$\%$ global stiffness $\mathrm{K}$

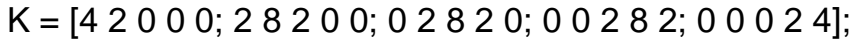

$f=Q^{*} a^{\wedge} 2$

$B=[f 1 ; f 2 ; f 3 ; f 4 ; f 5] ;$

$\mathrm{F}=\mathrm{B} \cdot{ }^{*} \mathrm{f}$

echo on

$\% \mathrm{~F}=\mathrm{K}^{*}$ theta, hence, theta $=\operatorname{inv}(\mathrm{K})^{\star} \mathrm{F}$

$\%$ theta $=[$ theta $1 ;$ theta $2 ;$ theta3; theta $4 ;$ theta5 $]$

echo off

theta $=\mathrm{K} \backslash \mathrm{F}$
\%For member forces (md)

$\% \mathrm{md}=[\mathrm{md} . . ; \mathrm{md} .$.

echo on

\%enter the values of terms of theta matrix above.

echo off

theta1 = input('theta1:');

theta2 = input('theta2:');

theta3 = input('theta3:');

theta4 = input('theta4:');

theta5 = input('theta5:');

thetaa $=[$ theta $1 ;$ theta2 $]$

thetab $=[$ theta2; theta3 $]$

thetac $=[$ theta3; theta 4$] ;$

thetad $=$ [theta4; theta5];

$\mathrm{md} 12=\mathrm{K} 1{ }^{*}$ thetaa

md23 $=\mathrm{K} 2{ }^{*}$ thetab

$\mathrm{md} 34=\mathrm{K} 3{ }^{*}$ thetac

$\mathrm{md} 45=\mathrm{K} 4{ }^{*}$ thetad

$\%$ Fixed end or edge moment

fem = [0; mxe; mxe1a; mxe1b; mxe2a; mxe2b; mxe3; 0];

echo on

\%Final Support moment(m)

echo off

$\% \mathrm{~m}=$ fem-md, $\mathrm{m} 12=$

fem12 = [0; mxe];

fem23 = [mxe1a; mxe1b];

fem34 = [mxe2a; mxe2b];

fem45 = [mxe3; 0];

$\mathrm{m} 12$ = fem12-md12

$\mathrm{m} 23=$ fem23-md23

m34 = fem34-md34

$\mathrm{m} 45$ = fem45-md45

$\%$ Average final Support moment (mavs) is mavs... = $(\mathrm{m} 21+\mathrm{m} 22) / 2$

echo on

\%input the positive values of $\mathrm{m}$ matrix above. note: $\mathrm{m} 12$

$=[\mathrm{ms} 11 ; \mathrm{ms} 21], \mathrm{m} 23=[\mathrm{m} 22 ; \mathrm{m} 32] \ldots$

echo off

ms11 = input('Enter moment at support 1 member 1:');

ms21 = input('Enter moment at support 2 member $1: ')$;

ms22 = input('Enter moment at support 2 member 2:');

ms32 = input('Enter moment at support 3 member 2:');

ms33 = input('Enter moment at support 3 member 3:');

ms43 = input('Enter moment at support 4 member 3:');

ms44 = input('Enter moment at support 4 member 4:');

ms54 = input('Enter moment at support 5 member 4:');

echo on

\%average final support moment

echo off

mavs $1=\mathrm{ms} 11$

mavs2 $=(\mathrm{ms} 21+\mathrm{ms} 22)^{\star} 0.5$

mavs $=(\mathrm{ms} 32+\mathrm{ms} 33)^{\star} 0.5$

mavs $4=(\mathrm{ms} 43+\mathrm{ms} 44)^{\star} 0.5$

mavs $5=\mathrm{ms} 54$

echo on

\%Span Moment(mspan)

echo off

$\%$ mspan $=0.125^{\star} Q^{*} a^{\wedge} 2-(m s t+m s t){ }^{*} 0.5$

$c=0.125^{\star} Q^{\star} a^{\wedge} 2$

mspan12 $=\mathrm{c}-\left((\text { mavs } 1+\text { mavs } 2)^{\star} 0.5\right)$

mspan23 $=c-\left((\text { mavs } 2+\text { mavs } 3)^{*} 0.5\right)$

mspan34 $=c-\left((\right.$ mavs3 + mavs 4$\left.){ }^{*} 0.5\right)$

mspan45

$=$

c- $\left((\text { mavs } 4+\text { mavs5 })^{\star} 0.5\right)$ 